\section{Optimizing Strategies for Immune Checkpoint Imaging with Immuno-PET in Preclinical Study}

TO THE EDITOR: Recently, we have read with interest the paper by Mayer et al. published in The Journal of Nuclear Medicine (1). The authors assessed the effects of 6 immuno-PET radiotracers on human programmed cell death ligand 1 (PD-L1) immune checkpoint imaging and discussed important design considerations that may affect biodistribution of radiotracers. Those radiotracers were specifically against human PD-L1 but did not cross-react with murine PD-L1. As we inferred, clinical immuno-PET tracers can bind not only to PD-L1 expressed by tumors, but also to PD-L1 expressed by normal cells. It is known that PD-L1 is expressed wildly on T cells, B cells, monocytes, and endothelial cells in both humans and mice (2). Therefore, radiotracer can be taken up by PD-L1-positive cells in organs, including lymphoid organs, lung, and liver, resulting in unexpected background signal and confounding determination of PD-L1 level in tumors. To optimize the immuno-PET imaging effect, especially in terms of background signal, we suggest using antimurine radiotracers and murine tumor cell lines for syngeneic tumor engraftments, because these will better fit the putative clinical status, rather than performing in vivo study in human tumor xenografts.

We are also concerned about the inherent characteristic of PDL1 after immuno-PET imaging. It is known that radiotracers can induce cell internalization; thus, the targeted receptor could be involved and relocated from membrane to cytoplasm (3,4). During immuno-PET imaging, PD-L1 is internalized but the metabolic mechanism is unclear, partially including degradation and repopulation back to the tumor cell surface. Moreover, whether the affinity between PD-L1 and tracer would change after being detected by immuno-PET for the first evaluation and monitoring assessment during treatment remains unknown. To identify the potential affinity change, we suggest conducting another immunoPET scan or surface plasmon resonance after the radiotracer is entirely eliminated.

Additionally, it is possible that the expression level of PD-L1 may not be a favorable biomarker for predicting anti-PD-L1 response. By analyzing the outcome of patients with different PD-L1 level, Robert et al. reported no difference in overall survival between the high-expression PD-L1 group and low or negative group after immunotherapy with anti-PD-L1 antibody (5). Therefore, high uptake of radiotracer at a tumor site may not predict a good response whereas low uptake may not indicate a poor response. To better predict anti-PD-L1 response, a combination of PD-L1 status and other cancer genetic biomarkers should be further considered (6).

Generally, immuno-PET imaging represents a novel imaging procedure and is helpful for selecting optimal patients and monitoring the expression status of specific molecules during anti-PD-L1 treatment. It could become the go-to complement to immunotherapy in the near future.

COPYRIGHT @ 2018 by the Society of Nuclear Medicine and Molecular Imaging.

\section{REFERENCES}

1. Mayer AT, Natarajan A, Gordon SR, et al. Practical immuno-PET radiotracer design considerations for human immune checkpoint imaging. J Nucl Med. 2017;58:538-546.

2. Sharpe AH, Wherry EJ, Ahmed R, Freeman GJ. The function of programmed cell death 1 and its ligands in regulating autoimmunity and infection. Nat Immunol. 2007;8:239-245.

3. Fani M, Mueller A, Tamma ML, et al. Radiolabeled bicyclic somatostatin-based analogs: a novel class of potential radiotracers for SPECT/PET of neuroendocrine tumors. J Nucl Med. 2010;51:1771-1779.

4. Varasteh Z, Velikyan I, Lindeberg G, et al. Synthesis and characterization of a high-affinity NOTA-conjugated bombesin antagonist for GRPR-targeted tumor imaging. Bioconjug Chem. 2013;24:1144-1153.

5. Robert C, Long GV, Brady B, et al. Nivolumab in previously untreated melanoma without BRAF mutation. $N$ Engl J Med. 2015;372:320-330.

6. Rosenberg JE, Hoffman-Censits J, Powles T, et al. Atezolizumab in patients with locally advanced and metastatic urothelial carcinoma who have progressed following treatment with platinum-based chemotherapy: a single-arm, multicentre, phase 2 trial. Lancet. 2016;387:1909-1920.

\author{
Jiale Chen \\ Ning Wang \\ Xinyu Yang \\ Yuan $\mathbf{L i}^{*}$
}

*Institute of Digestive Surgery State Key Laboratory of Biotherapy

West China Hospital

Sichuan University

No. 37, Guo Xue Lane

Chengdu 610041, Sichuan, China

E-mail: liyuanletters@163.com

Published online Nov. 16, 2017.

DOI: 10.2967/jnumed.117.204685

REPLY: In response to the comments made by Chen et al. regarding our paper "Practical Immuno-PET Radiotracer Design Considerations for Human Immune Checkpoint Imaging" published recently in The Journal of Nuclear Medicine (1), we have taken the opportunity to discuss several important points.

Chen et al. begin by suggesting the development of murine versus human radiotracers for testing in syngeneic models. In fact, this is a valuable suggestion, and our laboratory often develops and validates complementary murine and human radiotracers in parallel. An active area of investigation is the development of cross-reactive binders (with affinity for both human and murine targets), to help further streamline biologic characterization and clinical translation processes. That said, the greater debate here surrounds the question of model selection. Model selection is critical to the development of imaging agents, and the appropriate model should be chosen given the goals and hypotheses of the study at hand. Although the verdict is still out on the value of mouse models in drug development, we often use syngeneic models when our primary question pertains to the biology of the model system. Here, we used a human xenograft tumor model to assess and characterize the ability of our engineered tracer to bind specifically to human programmed cell death ligand 1 (PD-L1). This decision was made because our primary goal was toward clinical translation. Because of the rapid pace of immunotherapeutic drug development, we believe the imaging community must act quickly to 
assess the feasibility and benefit of companion diagnostic tools in clinical trials alongside the therapeutics they are meant to complement. Chen et al. rightly point out that PD-L1 is expressed by various other cell types, including immune cells. Although they are correct that this signal will have to be taken into consideration when assessing immune checkpoint therapy, it is important to remember that PD-L1-targeted drugs will also bind to these sites. We believe systems-level expression of PD-L1, not just tumor expression of PD-L1, will provide important insights into therapeutic success and potential drug toxicities. Choosing a model where we could validate that our binder was specifically binding to human PD-L1 was thus of critical importance for allowing us to eventually be able to interpret our signal in a human patient.

Concerns regarding the "inherent characteristic of PD-L1 after immuno-PET imaging" were also mentioned. What Chen et al. are likely alluding to here is the stability, internalization, and recycling of the complexed ligand receptor pair. Although we did not characterize these parameters in this work, they certainly all contribute in part to the PET signal at a given time and region of interest. In addition to studies to address tracer internalization, mathematic models can be used to assess bound versus unbound tracer in a target tissue, as well as estimate parameters, such as receptor density. These estimated model parameters can provide additional insight and enable more quantitative assessment than SUVs alone. Chen et al. discuss the potential of "affinity change" after in vivo administration of the immuno-PET probe. Although the affinity of the probe theoretically should not change barring breakdown of the protein, "blocking" can certainly affect longitudinal follow-up scans. Blocking occurs when cold probe or drug prevents binding of the radiolabeled agent. The risk of blocking is mitigated in this scenario by the low mass dose administration of the imaging agent, which should neither have pharmacologic effects nor saturate the binding sites. That said, we have discovered in previous work with antibody-based PET imaging agents that even low-dose administration can perturb the system (2), and thus it is an important concern to be investigated in all immuno-PET work moving forward.

Finally, Chen et al. suggest that PD-L1 may not be a favorable biomarker for predicting anti-PD-L1 response. Although there are some conflicting reports in the literature stemming in part from study design, sampling errors, and the currently available tools for measuring PD-L1 expression, there is a large body of evidence that suggests that PD-L1 expression does in fact correlate with therapeutic response to PD-L1 and PD-1-targeted antibodies (3). It is our hope that PET imaging of PD-L1 expression will help further elucidate responders versus nonresponders to therapy, as imaging is potentially better suited to the spatiotemporal varying expression patterns of immune checkpoint molecules. We agree with Chen et al. that combining immuno-PET imaging of PD-L1 with other biomarkers and tests will only likely improve our predictive accuracy.

Since our initial work on imaging PD-L1, many other groups have published papers on the subject (4-18). We strongly agree with Chen et al. that immuno-PET "could become the go-to complement to immunotherapy in the near future."

\section{REFERENCES}

1. Mayer AT, Natarajan A, Gordon SR, et al. Practical immuno-PET radiotracer design considerations for human immune checkpoint imaging. J Nucl Med. 2017;58:538-546.

2. James ML, Hoehne A, Mayer AT, et al. Imaging B cells in a mouse model of multiple sclerosis using ${ }^{64} \mathrm{Cu}$-Rituximab-PET. J Nucl Med. 2017;58:1845-1851.

3. Gandini S, Massi D, Mandalà M. PD-L1 expression in cancer patients receiving anti PD-1/PD-L1 antibodies: a systematic review and meta-analysis. Crit Rev Oncol Hematol. 2016;100:88-98.

4. Donnelly DJ, et al. Synthesis and biological evaluation of a novel ${ }^{18} \mathrm{~F}$-labeled adnectin as a PET radioligand for imaging PD-L1 expression. J Nucl Med. October 12, 2017 [Epub ahead of print].

5. Chatterjee S, Lesniak W, Nimmagadda S. Noninvasive imaging of immune checkpoint ligand PD-L1 in tumors and metastases for guiding immunotherapy. Mol Imaging. 2017; 16:1536012117718459.

6. Broos K, Keyaerts M, Lecocq Q, et al. Non-invasive assessment of murine PDL1 levels in syngeneic tumor models by nuclear imaging with nanobody tracers. Oncotarget. 2017;8:41932-41946.

7. Hettich M, Braun F, Bartholomä MD, Schirmbeck R, Niedermann G. High-resolution PET imaging with therapeutic antibody-based PD-1/PD-L1 checkpoint tracers. Theranostics. 2016;6:1629-1640.

8. Josefsson A, Nedrow JR, Park S, et al. Imaging, biodistribution, and dosimetry of radionuclide-labeled PD-L1 antibody in an immunocompetent mouse model of breast cancer. Cancer Res. 2016;76:472-479.

9. Chatterjee S, Lesniak WG, Gabrielson M, et al. A humanized antibody for imaging immune checkpoint ligand PD-L1 expression in tumors. Oncotarget. 2016;7:10215-10227.

10. Nedrow JR, Josefsson A, Park S, Ranka S, Roy S, Sgouros G. Imaging of programmed cell death ligand 1: impact of protein concentration on distribution of anti-PD-L1 SPECT agents in an immunocompetent murine model of melanoma. J Nucl Med. 2017;58:1560-1566.

11. González Trotter DE, Meng X, McQuade P, et al. In vivo imaging of the programmed death ligand 1 by ${ }^{18} \mathrm{~F}$ positron emission tomography. $\mathrm{J} \mathrm{Nucl}$ Med. 2017;58:1852-1857.

12. Lesniak WG, Chatterjee S, Gabrielson M, et al. PD-L1 detection in tumors using $\left[{ }^{64} \mathrm{Cu}\right]$ Atezolizumab with PET. Bioconjug Chem. 2016;27:2103-2110.

13. Heskamp S, Hobo W, Molkenboer-Kuenen JD, et al. Noninvasive imaging of tumor PD-L1 expression using radiolabeled anti-PD-L1 antibodies. Cancer Res. 2015;75:2928-2936.

14. Kikuchi M, Clump DA, Srivastava RM, et al. Preclinical immunoPET/CT imaging using Zr-89-labeled anti-PD-L1 monoclonal antibody for assessing radiation-induced PD-L1 upregulation in head and neck cancer and melanoma. OncoImmunology. 2017;6:e1329071.

15. Truillet $\mathrm{C}$, Oh HLJ, Yeo SP, et al. Imaging PD-L1 expression with ImmunoPET. Bioconjug Chem. 2018;29:96-103.

16. Meir R, Shamalov K, Sadan T, et al. Fast image-guided stratification using antiprogrammed death ligand 1 gold nanoparticles for cancer immunotherapy. ACS Nano. 2017;11:11127-11134.

17. Chatterjee S, Lesniak WG, Miller MS, et al. Rapid PD-L1 detection in tumors with PET using a highly specific peptide. Biochem Biophys Res Commun. 2017; 483:258-263.

18. Maute RL, Gordon SR, Mayer AT, et al. Engineering high-affinity PD-1 variants for optimized immunotherapy and immuno-PET imaging. Proc Natl Acad Sci USA. 2015;112:E6506-E6514.

\author{
Aaron T. Mayer \\ Sanjiv S. Gambhir* \\ *Stanford University \\ James H. Clark Center \\ 318 Campus Dr., E153 \\ Stanford, CA 94305 \\ E-mail: sgambhir@stanford.edu
}

Published online Jan. 18, 2018.

DOI: 10.2967/jnumed.117.205153 